\title{
Charge expulsion and electric field in superconductors
}

\author{
J. E. Hirsch \\ Department of Physics, University of California, San Diego \\ La Jolla, CA 92093-0319
}

(Dated: August 31, 2003)

\begin{abstract}
The theory of hole superconductivity predicts that when a metal goes superconducting negative charge is expelled from its interior towards the surface. As a consequence the superconductor in its ground state is predicted to have a non-homogeneous charge distribution and an outward pointing electric field in its interior. Here we propose equations to describe the behavior of the charge density and electric field in superconductors, and solve them for a spherical geometry. The magnitude of the predicted interior electric field depends on superconducting parameters such as the condensation energy and the London penetration depth and is found to be of order $10^{6} \mathrm{~V} / \mathrm{cm}$. A physical interpretation of the result is given. It is predicted that for small superconducting bodies (compared to the penetration depth) an electric field outside the superconductor should result from this physics. This may explain a recent experimental observation in $N b$ metal clusters.
\end{abstract}

PACS numbers:

\section{INTRODUCTION}

In the currently accepted understanding of superconductivity no electric fields exist in superconductors in the absence of electric current (a 'Bernoulli potential' is expected to exist in the presence of a non-uniform supercurrent [1]). The London brothers [2] originally proposed a set of equations to describe superconductivity that allowed for the presence of an electric field within a penetration depth of the surface of a superconductor. However H. London failed to detect such an electric field experimentally [3]. Having confidence in his brother's experimental result, F. London modified the theory and discarded one of their original equations, and as a consequence the possibility of an electric field in superconductors is no longer discussed in London's definitive work [4].

As is well known, normal metals allow for the existence of magnetic fields but no electric fields in their interior. Superconductors (type I) do not allow magnetic fields in their interior, but magnetic fields can exist within a penetration depth of the surface. Based on the theory of hole superconductivity 5, 6] we have recently proposed that when a metal goes superconducting negative charge is expelled from its interior towards the surface [7]. Here we discuss the equations governing the charge and electric field distribution in superconductors resulting from this physics. The possibility that a superconductor may have an electric field in its interior has not been discussed in other theoretical frameworks to our knowledge; it is however a necessary consequence of the fundamental electron-hole asymmetry of condensed matter [8], and the resulting 'giant atom' description of superconductors [9] that results from the theory of hole superconductivity.

\section{ENERGETICS}

The qualitative picture of a superconductor proposed in refs. [7, 9] is shown in figure 1: it looks like a gi-

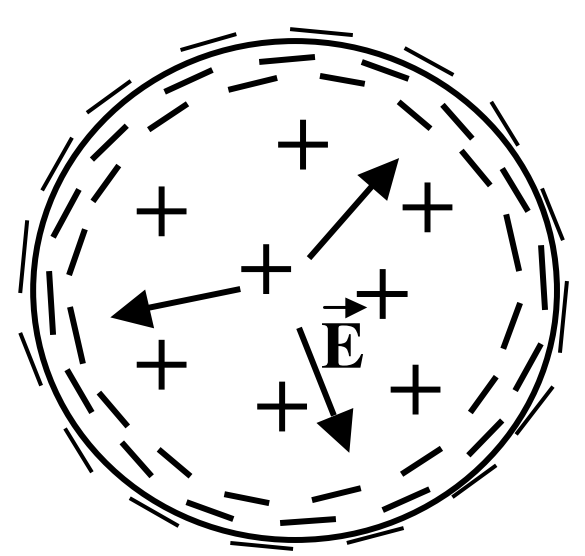

FIG. 1: Schematic picture of a spherical superconducting body. Negative charge is expelled from the bulk to the surface. As a consequence, an outward-pointing electric field exists in the interior.

ant atom, with a higher density of negative charge near the surface and higher density of positive charge in the interior 10]. Consider a superconducting sphere of radius $R$ much larger than its London penetration depth $\lambda_{L}$. Assume an amount of charge $q$ moves from the interior to the surface, resulting in a uniform charge distribution $\rho_{0}$ in the interior:

$$
\rho_{0}=-\frac{3 q}{4 \pi R^{3}}
$$

As we discuss later, $q$ is negative and resides within a London penetration depth of the surface, with volume charge density

$$
\rho_{-}=\frac{q}{4 \pi R^{2} \lambda_{L}}=-\frac{1}{3} \frac{R}{\lambda_{L}} \rho_{0}
$$


For $\lambda_{L}<<R$ we can also think of this charge as a 'surface charge density'

$$
\sigma=\frac{q}{4 \pi R^{2}}=\rho_{-} \lambda_{L}
$$

This 'surface charge density' is not confined within a Thomas Fermi screening length of the surface as excess charge would be in ordinary metals, but within the much thicker surface layer defined by the penetration depth.

The electric field in the interior is given by

$$
\vec{E}(r)=-\frac{q}{R^{3}} \vec{r}
$$

and the inhomogeneous charge distribution gives rise to a cost in Coulomb energy

$$
U_{E}=\int_{0}^{R} d^{3} r \frac{E(r)^{2}}{8 \pi}=\frac{q^{2}}{10 R}
$$

under the assumption $\lambda_{L}<<R$. For a spherical geometry no electric field exists outside the superconductor (assuming charge neutrality).

In the superconducting state, the energy per unit volume is lowered by the superconducting condensation energy. The theory of hole superconductivity predicts that the charge expulsion describe above is a necessary consequence of superconductivity. Hence the Coulomb energy cost Eq. (5) is balanced by an energy gain due to the charge expulsion, $\tilde{\epsilon}$ per unit volume, which is related but not necessarily equal to the condensation energy of the superconductor. Equating this energy gain to the electrostatic energy cost,

$$
\frac{q^{2}}{10 R}=\frac{4}{3} \pi R^{3} \tilde{\epsilon}
$$

yields for the 'surface charge density'

$$
\sigma=\left[\frac{5}{6 \pi} \tilde{\epsilon}\right]^{1 / 2}
$$

independent of the sphere's radius. This indicates that if the physics discussed here indeed exists in superconductors, the charge density in the surface layer $\rho_{-}$is an intensive quantity determined by intrinsic properties of the superconductor, in particular the penetration depth and the thermodynamic critical field $H_{c}$ (which gives the condensation energy). Instead, the interior positive charge density $\rho_{0}$ is not intensive but decreases with increasing radius $R$. The electric field close to the surface, given by Eq. (4) for $r \sim R$, is also independent of the body's dimensions.

From Eqs. (3) and (7) the volume charge density in the surface layer is given by

$$
\rho_{-}=-\left[\frac{5}{6 \pi \lambda_{L}^{2}} \tilde{\epsilon}\right]^{1 / 2}
$$

Using the expression for the London penetration depth

$$
\frac{1}{\lambda_{L}^{2}}=\frac{4 \pi n_{s} e^{2}}{m_{e} c^{2}}
$$

with $m_{e}$ the free electron mass, $e$ the electron charge (negative) and $n_{s}$ the superfluid density per unit volume, and replacing $\tilde{\epsilon}$ in terms of the energy gained per superfluid electron $\epsilon$ yields

$$
\epsilon=\frac{\tilde{\epsilon}}{n_{s}}
$$

and Eq. (8) becomes

$$
\rho_{-}=e n_{s}\left(\frac{10}{3} \frac{\epsilon}{m_{e} c^{2}}\right)^{1 / 2}
$$

relating the negative excess charge density in the surface layer to the superfluid density $n_{s}$ and the ratio of the condensation energy of an electron $\epsilon$ to its rest energy, showing that the excess charge density is a small fraction of the superfluid density. The 'surface charge density' $\sigma$ is given by Eq. (3), and the maximum electric field attained within a penetration length of the surface of the superconductor is

$$
E_{\max }=-4 \pi \sigma=-4 \pi \lambda_{L} \rho_{-}
$$

and is also independent of the sample's dimensions.

As an example appropriate for high $T_{c}$ cuprates we take $\lambda_{L}=2000 \AA$, yielding from Eq. (9) a superfluid density $n_{s}=7.1 \times 10^{-4}$ electrons $/ \AA^{3}$. Assuming a condensation energy of $100 \mu \mathrm{eV}$ per unit cell and a unit cell volume of $40 \AA^{3}$ yields $\epsilon=3.5 \mathrm{meV}$ per superfluid electron, and $\rho_{-}=-1.5 \times 10^{-4} n_{s} e$ so there is roughly 1 extra electron per 10,000 superfluid electrons in the outer layer. The 'surface charge density' is $\sigma=-3.5 \times 10^{-7} \mathrm{C} / \mathrm{cm}^{2}$, and the maximum electric field near the surface is $E_{\max }=$ $3.9 \times 10^{6} \mathrm{~V} / \mathrm{cm}$. The potential difference between the center of the sphere and a point within a penetration depth of the surface is $\Delta V=E_{\max } R / 2$, so approximately 4 million Volt for a sample of $1 \mathrm{~cm}$ radius.

As another example, for $\mathrm{Nb}$ the thermodynamic critical field is $H_{c}=1980 G$, so the condensation energy is $\tilde{\epsilon}=1.56 \times 10^{5} \mathrm{ergs} / \mathrm{cm}^{3}$. From Eq. (7) the 'surface charge density' is $\sigma=6.8 \times 10^{-8} \mathrm{C} / \mathrm{cm}^{2}$ and taking $\lambda_{L}=400 \AA$ for the penetration depth yields $\rho_{-}=0.017 \mathrm{C} / \mathrm{cm}^{3}$ for the surface layer volume charge density, corresponding to approximately 2 excess electrons per million $\mathrm{Nb}$ atoms in the outer layer. The maximum electric field near the surface from Eq. (12) is $E_{\max }=0.77 \times 10^{6} \mathrm{~V} / \mathrm{cm}$. This is not very different from the estimate for the high $T_{c}$ case because the effects of a smaller condensation energy and a smaller London penetration depth partially compensate each other.

\section{ELECTRIC FIELD EQUATIONS}

We start with the London equation for the supercurrent

$$
\vec{J}=-\frac{n_{s} e^{2}}{m_{e} c} \vec{A}
$$


with $\vec{A}$ the magnetic vector potential. Following the Londons [2] we assume that $\vec{A}$ satisfies the Lorenz gauge condition

$$
\vec{\nabla} \cdot \vec{A}+\frac{1}{c} \frac{\partial \phi}{\partial t}=0
$$

with $\phi$ the electric potential. The electric field is given by

$$
\vec{E}=-\vec{\nabla} \phi-\frac{1}{c} \frac{\partial \vec{A}}{\partial t}
$$

Using the continuity equation

$$
\vec{\nabla} \cdot \vec{J}=-\frac{\partial \rho}{\partial t}
$$

and applying the divergence operator to both sides of Eq. (13) and using the gauge condition Eq. (14) yields

$$
\frac{\partial \phi}{\partial t}=-\frac{m_{e} c^{2}}{n_{s} e^{2}} \frac{\partial \rho}{\partial t}
$$

hence

$$
\phi(\vec{r}, t)=-\frac{m_{e} c^{2}}{n_{s} e^{2}} \rho(\vec{r}, t)+\phi_{0}(\vec{r})
$$

The London brothers postulated Eq. (18) with $\phi_{0}(\vec{r})=0$ as a possible equation applicable to superconductors 2]. Under stationary conditions,

$$
\phi(\vec{r})=-4 \pi \lambda_{L}^{2} \rho(\vec{r})
$$

and using Maxwell's equation

$$
\vec{\nabla} \cdot \vec{E}(\vec{r})=4 \pi \rho(\vec{r})
$$

Eqs. (15) and (19) yield

$$
\begin{aligned}
& \rho(\vec{r})=\lambda_{L}^{2} \nabla^{2} \rho(\vec{r}) \\
& \vec{E}(\vec{r})=\lambda_{L}^{2} \nabla^{2} \vec{E}(\vec{r})
\end{aligned}
$$

which imply that a non-zero charge density and an electric field can exist within a penetration depth of the surface of the superconductor but not in the interior. $\mathrm{H}$. London attempted to measure the electric field predicted by Eq. (21) by measuring the change in capacitance of a capacitor with superconducting plates when it is cooled below $T_{c}[\underline{3}$. However he failed to detect any effect.

Here we propose that Equation (18) is valid for superconductors with a non-zero $\phi_{0}(r)$ resulting from a uniform charge density $\rho_{0}$ deep in the interior of the superconductor, as discussed in the previous section. Eqs. (21) and (18) then become

$$
\rho(r)=\rho_{0}+\lambda_{L}^{2} \nabla^{2} \rho(r)
$$

$$
\begin{aligned}
& \vec{E}(r)=\vec{E}_{0}(r)+\lambda_{L}^{2} \nabla^{2} \vec{E}(r) \\
& \phi(\vec{r})=-4 \pi \lambda_{L}^{2} \rho(\vec{r})+\phi_{0}(\vec{r})
\end{aligned}
$$

with $\vec{E}_{0}(\vec{r})=-\vec{\nabla} \phi_{0}(\vec{r})$ and $\vec{\nabla} \cdot \vec{E}_{0}(\vec{r})=4 \pi \rho_{0}$. We propose that these equations hold for the interior of superconductors of arbitrary shape in the absence of magnetic fields and electric currents, with $\rho_{0}$ a positive number determined by the microscopic parameters of the superconductor as well as the geometry, as discussed in the previous section. The potential obeys

$$
\phi(\vec{r})=\phi_{0}(\vec{r})+\lambda_{L}^{2} \nabla^{2} \phi(\vec{r})
$$

$$
\nabla^{2} \phi_{0}(\vec{r})=-4 \pi \rho_{0}
$$

in the interior, and

$$
\begin{gathered}
\nabla^{2} \phi(\vec{r})=0 \\
\nabla^{2} \phi_{0}(\vec{r})=0
\end{gathered}
$$

in the exterior of the body. Furthermore we assume no surface charges can exist in the superconductor, hence that both $\phi$ and its normal derivative $\partial \phi / \partial n$ are continuous on the surface of the body, as are of course $\phi_{0}$ and $\partial \phi_{0} / \partial n$.

Note that the existence of an electric field in the interior of the superconductor does neither imply a time variation of the current, as one would expect in a 'perfect conductor', nor even the existence of a current as in an ordinary metal. Taking the time derivative of Eq. (13) and using the Maxwell equation (15) yields

$$
\frac{\partial \vec{J}}{\partial t}=\frac{n_{s} e^{2}}{m}(\vec{\nabla} \phi+\vec{E})
$$

so that in a stationary situation there can be an electric field that is derivable from a potential $(\vec{E}=-\vec{\nabla} \phi)$ and it does not lead to a time-varying supercurrent. Whether a stationary supercurrent exists or not depends on the magnetic vector potential $\vec{A}$ through Eq. (13) and not on the electric field.

In a spherical geometry, the solution of Eq. (22a) is

$$
\rho(r)=\rho_{0}-k \frac{\sinh \left(r / \lambda_{L}\right)}{r}
$$

and for charge neutrality the constant $k$ is given by

$$
k=\frac{1}{3} \frac{\rho_{0} R^{3}}{\lambda_{L}^{2}} \frac{1}{f\left(R / \lambda_{L}\right)}
$$



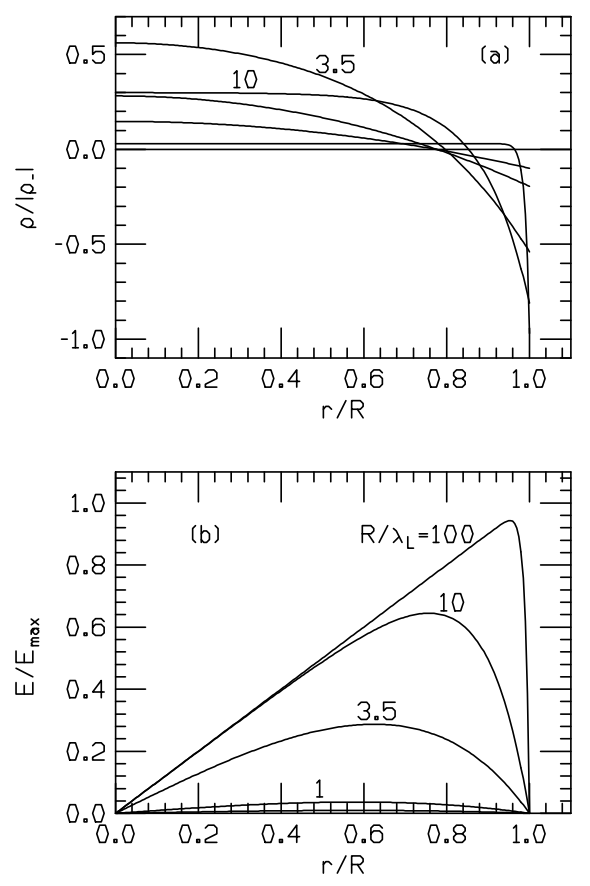

FIG. 2: Radial dependence of charge density (a) and electric field (b) for $R / \lambda_{L}=0.5,1,3.5,10$ and 100 (some numbers next to the lines). The electric field increases monotonically with increasing $R / \lambda_{L}$. The charge density at the origin is smallest for $R / \lambda_{L}=100$, and decreases with decreasing $R / \lambda_{L}$ for $R / \lambda_{L}<3.5$.

$$
f(x)=x \cosh x-\sinh x
$$

For $R>>\lambda_{L}$ the charge density at the surface is

$$
\rho(R)=\rho_{0}\left(1-\frac{1}{3} \frac{R}{\lambda_{L}}\right) \sim-\frac{R}{3 \lambda_{L}} \rho_{0}
$$

in accordance with Eqs. (1), (2), and for $r<<R-\lambda_{L}$, $\rho(r) \sim \rho_{0}$. The electric field is given by

$$
\vec{E}(r)=\frac{4}{3} \pi \rho_{0}\left[1-\frac{R^{3}}{r^{3}} \frac{f\left(r / \lambda_{L}\right)}{f\left(R / \lambda_{L}\right)}\right] \vec{r}
$$

It goes to zero at $r=R$ and is maximum at a distance of a penetration depth inside the surface, given by

$$
E_{\text {max }} \sim \frac{4}{3} \pi \rho_{0} R=-4 \pi \lambda_{L} \rho(R)
$$

in agreement with Eq. (12) for $\rho(R)=\rho_{-}$, as expected.

Figure 2 shows the radial dependence of the charge density and electric field for various values of $R / \lambda_{L}$. When the dimensions of the sample are much larger than the penetration depth there is a small uniform positive charge density in the interior and the electric field increases linearly towards the surface. The compensating negative charge resides within a penetration length of the surface and the electric field drops to zero in that region. As the dimensions of the sample decrease the positive charge density in the interior increases and is maximum for $R / \lambda_{L} \sim 3.5$, where $\rho_{0} \sim-0.56 \rho_{-}$, and decreases again for even smaller samples. This is under the assumption that superconducting properties are not affected by the size of the sample, i.e. $\rho_{-}$and $\lambda_{L}$ are assumed independent of $R$. In the limit $R / \lambda_{L}<<1$ the charge density is given by

$$
\rho(r)=\frac{\rho_{0}}{6 \lambda_{L}^{2}}\left[\frac{3}{5} R^{2}-r^{2}\right]
$$

so that it changes sign at $r / R=0.77$, and the electric field by

$$
\vec{E}(\vec{r})=\frac{2 \pi}{15} \frac{\rho_{0}}{\lambda_{L}^{2}}\left[R^{2}-r^{2}\right] \vec{r} .
$$

\section{MICROSCOPIC INTERPRETATION}

The existence of an electric field in the interior of a superconductor may seem surprising. In normal metals, electric fields cannot exist in the interior because the mobile electronic charge will rearrange to nullify such field. How can the existence of an electric field in a superconductor be understood?

Consider Eq. (24). It states that no electric current will be generated in the presence of an interior electric field, provided the field derives from a potential $\phi$. Now an electric field exerts a force on electrons, why is it that electrons in the superconductor do not get accelerated by the interior electric field and generate an electric current?

The Lorentz force equation in the absence of a magnetic field

$$
m_{e} \frac{d \vec{v}}{d t}=e \vec{E}
$$

indicates that the velocity of an electron will change in the presence of an electric field. From the relation between velocity and current

$$
\vec{J}=e n_{s} \vec{v}
$$

one may be tempted to conclude that Eqs. (32) and (33) are incompatible with Eq. (24). However this is not the case, because Eq. (24) is a local time derivative and Eq. (32) is a total time derivative. Using the relation[11]

$$
\frac{d \vec{v}}{d t}=\frac{\partial \vec{v}}{\partial t}+\vec{\nabla} \frac{v^{2}}{2}-\vec{v} \times(\vec{\nabla} \times \vec{v})
$$

we conclude that a stationary situation with an electric field in the interior of the superconductor is indeed possible, provided that

$$
\vec{E}=\frac{m_{e}}{e}\left[\vec{\nabla} \frac{v^{2}}{2}-\vec{v} \times(\vec{\nabla} \times \vec{v})\right]
$$


If we assume that the superfluid electrons traverse circular orbits with speed $v(r)$ we have

$$
\vec{\nabla} \frac{v^{2}}{2}-\vec{v} \times(\vec{\nabla} \times \vec{v})=-\frac{v^{2}(r)}{r} \hat{r}
$$

and the speed is obtained from Eqs. (35) and (28) as

$$
v^{2}(r)=-\frac{4}{3} \frac{\pi e \rho_{0}}{m_{e}} r^{2}\left[1-\frac{R^{3}}{r^{3}} \frac{f\left(r / \lambda_{L}\right)}{f\left(R / \lambda_{L}\right)}\right]
$$

which of course simply describes the balance between centripetal acceleration and electric Lorentz force

$$
\frac{m_{e} v^{2}}{r}=-e E
$$

as appropriate for a circular orbit. Deep in the interior of the superconductor Eq. (37) describes a 'rigid rotation' of the superfluid, $v(r)=\omega r$, with constant angular velocity

$$
\omega=\left(-\frac{4 \pi \rho_{0} e}{3 m_{e}}\right)^{1 / 2}
$$

As we approach the surface the angular velocity decreases to zero due to the screening of the electric field by the negative charge in the surface layer.

We conclude from this interpretation that the 'rigidity' of the wave function [4] that allows for an electric field in the interior arises from the fact that superfluid electrons traverse macroscopic orbits at high speed; a radial electric field will not change the radial speed of electrons for the same reason that an orbiting planet is not attracted towards the sun: the radial force provides the centripetal acceleration for the orbital motion.

However one may also ask: if there is a finite velocity as given by Eq. (37), shouldn't there be a finite current and an associated magnetic field? In fact Eq. (33) is an oversimplification, which assumes that all electrons at position $\vec{r}$ move with the same velocity $\vec{v}$. However electrons also have a spin quantum number. As the simplest resolution to this question we may assume that the velocity field for spin direction $\sigma$ (with respect to a chosen quantization axis) obeys

$$
\vec{v}_{\sigma}(\vec{r})=-\vec{v}_{-\sigma}(\vec{r})
$$

with $v_{\sigma}$ given by Eq. (37). In that case the total charge current

$$
\vec{J}=e \frac{n_{s}}{2}\left(\vec{v}_{\uparrow}+\vec{v}_{\downarrow}\right)
$$

will be zero and no magnetic field will be generated, however there will be a spin current

$$
\vec{J}_{\text {spin }}=e \frac{n_{s}}{2}\left(\vec{v}_{\uparrow}-\vec{v}_{\downarrow}\right)
$$

which is not zero. The direction of the spin current would be determined by spin-orbit coupling as discussed in Ref. 9].
A different simple solution of Eq. (35) is obtained under the assumption $\vec{\nabla} \times \vec{v}=0$. In that case, we have simply

$$
\vec{E}=\frac{m_{e}}{2 e} \vec{\nabla} v^{2}
$$

and the velocity profile is readily obtained by integration of Eq. (28) as

$$
v^{2}(r)=\frac{4}{3} \frac{\pi e \rho_{0}}{m_{e}} r^{2}\left[1-2 \frac{R^{3}}{r^{3}} \frac{\sinh \left(r / \lambda_{L}\right)}{f\left(R / \lambda_{L}\right)}\right]+k
$$

with $k$ an integration constant. Deep in the interior of the superconductor we have (for $R / \lambda_{L}>>1$ )

$$
v^{2}=\frac{4 \pi \rho_{0} e}{3 m_{e}} r^{2}+k
$$

Eq. (44b) has a simple physical interpretation. The potential in which the electron moves in the interior of the superconductor is a harmonic oscillator potential:

$$
U(r)=e \phi_{0}(r)=-\frac{2}{3} \pi \rho_{0} e r^{2}
$$

where $\phi_{0}(r)$ follows from Eq. (23b). The total energy of the electron is sum of kinetic and potential energy:

$$
E_{t o t}=\frac{1}{2} m_{e} v^{2}-\frac{2}{3} \pi \rho_{0} e r^{2}
$$

and is a constant of motion. Indeed, Eq. (46) is the same as Eq. (44b), with the integration constant $k=2 E_{\text {tot }} / m_{e}$. The allowed values of $k$ are such that the maximum elongation $r_{\max }$ for which $v$ goes to zero obeys $r_{\max } \leq R$. When $k$ is such that $r_{\max }$ approaches $R$, the motion is slightly faster than in the harmonic oscillator potential near the region of maximum elongation (as given by Eq. (44a)), due to screening by the negative charge near the surface. For small $R / \lambda_{L}$ instead the velocity is very different than Eq. (44b) and is given by

$$
v^{2}=k^{\prime}+\frac{2 \pi e \rho_{0}}{15 m_{e} \lambda_{L}^{2}} r^{2}\left[R^{2}-\frac{r^{2}}{2}\right]
$$

Note also that an electron that moves in a harmonic oscillator potential does not give rise to uniform charge density, but rather the electron density and hence negative charge is largest near the region of maximum classical elongation. This is consistent with the assumption that the charge density in the superconductor is positive in the interior and negative near the surface. Similarly, circular 'Bohr orbits' in a harmonic oscillator potential 'bunch up' for large radius as discussed in Ref. [9] giving rise to larger negative charge density near the surface.

The assumption $\vec{\nabla} \times \vec{v}=0$ implies linear motion where the electrons oscillate through the origin. Relaxing this condition many other trajectories become possible. Deep in the interior the motion is simply described by independent harmonic motion in the three directions with 
frequency given by Eq. (39), which in general describes elliptical orbits. The linear oscillator and circular motion described above are two particular examples of these orbits where it is simple to obtain the corrections due to the outer layer of negative charge, as given by Eqs. (44a) and (37).

\section{SUPERCONDUCTORS OF GENERAL SHAPE}

From Eq. (22a) we have

$$
\tilde{\rho}(\vec{r})=\lambda_{L} \nabla^{2} \tilde{\rho}(\vec{r})
$$

for $\tilde{\rho}(\vec{r})=\rho(\vec{r})-\rho_{0}$. Laue has shown rigurously 12 that for a body of arbitrary shape the solution of Eq. (48) decays to zero rapidly towards the interior, on the length scale of the penetration depth $\lambda_{L}$. Hence we can conclude that quite generally for superconductors of dimensions much larger than $\lambda_{L}$ the charge distribution in the interior is constant and equal to $\rho_{0}$, and that the expelled negative charge resides within $\lambda_{L}$ of the surface.

Similarly using that

$$
\vec{\nabla} \times\left(\vec{\nabla} \times \vec{E}_{0}\right)=\vec{\nabla}\left(\vec{\nabla} \cdot \vec{E}_{0}\right)-\nabla^{2} \vec{E}_{0}
$$

as well as $\vec{\nabla} \times \vec{E}_{0}=0$ and $\vec{\nabla} \cdot \vec{E}_{0}=$ constant we conclude that $\nabla^{2} \vec{E}_{0}=0$, hence from Eq. (22b)

$$
\nabla^{2}\left(\vec{E}-\vec{E}_{0}\right)=\frac{1}{\lambda_{L}^{2}}\left(\vec{E}-\vec{E}_{0}\right)
$$

This equation is the same as the London equation satisfied by the magnetic field in non-rotating superconductors, giving rise to the Meissner effect. Again, Laue showed [12] that the vector quantity satisfing Eq. (50) decays rapidly to zero over a distance of a penetration depth from the surface. Hence we conclude that for superconductors of arbitrary shape away form the surface the electric field is given by $\vec{E}_{0}$ originating in the uniform charge distribution $\rho_{0}$. For example, for an ellipsoid of revolution with symmetry axis along the $z$ direction

$$
\frac{x^{2}+y^{2}}{a^{2}}+\frac{z^{2}}{b^{2}}=1
$$

the electric field is given by 14

$$
\begin{gathered}
\left(E_{0}\right)_{x}=\frac{2}{3} \pi \rho_{0}(3-p) \frac{x}{a^{2} b} \\
\left(E_{0}\right)_{z}=\frac{4}{3} \pi \rho_{0} p \frac{x}{a^{2} b}
\end{gathered}
$$

with $p=b / a$. Eq. (52) is approximately valid for $0.8<$ $p<5$. In particular, the surface of a uniformly charged ellipsoid is not an equipotential surface, and this is of course also the case for a body of arbitrary non-spherical shape.
In the presence of the expelled negative charge we argue that in general the surface of the body will still not be an equipotential surface, and hence that electric fields will exist outside the superconductor. This is seen as follows: we define

$$
\tilde{\phi}(\vec{r})=\phi(\vec{r})-\phi_{0}(\vec{r})+4 \pi \lambda_{L}^{2} \rho_{0}
$$

which obeys the differential equation

$$
\tilde{\phi}(\vec{r})=\lambda_{L}^{2} \nabla^{2} \tilde{\phi}(\vec{r})
$$

inside the body, and

$$
\nabla^{2} \tilde{\phi}(\vec{r})=0
$$

outside. Furthermore, both $\tilde{\phi}$ and its normal derivative $\partial \tilde{\phi} / \partial n$ are continuous on the surface of the body. The function $\tilde{\phi}$ satisfying the differential equation (54) is uniquely determined by specifying either $\tilde{\phi}$ or $\partial \tilde{\phi} / \partial n$ on the surface of the body. For Eq. (54b) this is well known [13], for Eq. (54a) it follows similarly from Green's theorem since for any two solutions of Eq. (54a) with the same boundary values their difference $U(\vec{r})$ satisfies

$$
\int_{V} d^{3} r\left[|\vec{\nabla} U|^{2}+\frac{U^{2}}{\lambda_{L}^{2}}\right]=\oint_{S} U \frac{\partial U}{\partial n} d a
$$

hence $U$ is identically zero in the interior. If no electric field existed outside we would have $\phi=$ constant and $\partial \phi / \partial n=0$ on the surface of the body, and the function $\tilde{\phi}$ would be overdetermined, i.e. there is in general no solution to the differential equation (54) with both $\tilde{\phi}$ and $\partial \tilde{\phi} / \partial n$ specified on a closed surface.

To solve the problem in general numerically we suggest the following procedure. Assume an initial guess for $\phi(\vec{r})$ on the surface (for example constant). Solve the Dirichlet problem for $\tilde{\phi}$ inside and outside, and find $\partial \tilde{\phi} / \partial n$ at the surface coming from the inside and the outside, which in general will not match. Hence $\partial \phi / \partial n$ is discontinuous at the surface, which implies the presence of a surface charge density

$$
\left.\left.\sigma=\frac{1}{4 \pi}\left[\frac{\partial \phi}{\partial n}\right)_{\text {inside }}-\frac{\partial \phi}{\partial n}\right)_{\text {outside }}\right]
$$

Since we assume no such surface charge is possible in superconductors it indicates that the initial guess for $\phi(\vec{r})$ on the surface was incorrect. We next compute the average of $\partial \phi / \partial n$ inside and outside the surface, and solve the corresponding Neumann problem for that boundary condition inside and outside. The resulting $\phi(\vec{r})$ on the surface will in general be discontinuous, so we calculate its average coming from inside and outside and solve again the Dirichlet problem with the new boundary condition. Thus solving a sequence of alternating Dirichlet and Neumann problems the solution should converge to a unique solution with both $\phi$ and $\partial \phi / \partial n$ continuous at the surface. 
These considerations also indicate that for superconductors of dimensions much larger than the penetration depth the negative charge near the surface will arrange laterally so as to nullify any tangential electric field and hence that the surface of the body will be very nearly an equipotential surface. This is because the surface charge density Eq. (56) required for it can be achieved by arranging the charge in the surface layer of thickness $\lambda_{L}$ appropriately. In other words, the solution to the differential equations where the surface charge $\sigma$ in Eq. (56) is spread out over the layer of thickness $\lambda_{L}$ and $\phi$ is very nearly constant on the surface will be the unique solution for a body of dimensions much larger than $\lambda_{L}$. If $\phi$ is constant on the surface and the body is charge neutral, no electric field lines can exist outside: for any exterior electric field line starting on the surface would have to return to another point on the surface, and the integral of the electric field along that line would yield a difference in potential at the initial and final points, both on the surface. Hence we conclude that for a charge neutral superconductor of large dimensions essentially no electric field lines will exist outside the superconductor, and it is not possible to detect the non-uniform charge distribution in the interior by measurements outside the superconductor.

Instead, for superconductors of dimensions comparable to or smaller than the penetration depth the negative charge distribution cannot be regarded as a 'surface charge', and it will not cancel the potential differences at different points of the surface originating in the positive charge. Hence the electric field will 'leak out' to the exterior of the superconductor and become observable.

The simplest shape that can give rise to such effect is an ellipsoid of revolution, described by Eq. (51). For a uniformly charged ellipsoid with charge density $\rho_{0}$ the dipole moment (with respect to the center of mass) is zero, but the quadrupole moment is given by

$$
Q \equiv \int d^{3} r \rho(\vec{r})\left(3 z^{2}-r^{2}\right)=\frac{8}{15} \pi a^{2} b\left(b^{2}-a^{2}\right) \rho_{0}
$$

For the charge neutral superconductor, if negative charge is expelled towards the surface the charge distribution looks qualitatively as in Figure 3. If the dimensions of the body are not much larger than the penetration depth it is plausible to assume that the negative charge will not rearrange laterally but instead will remain approximately uniform. It is clear from Fig. 3 that the negative charge will give a larger contribution to the quadrupole moment than the positive charge. Consequently this will give rise to a net negative quadrupole moment for a prolate ellipsoid ( $b>a$ with $b$ in the $z$ direction), and a net positive quadrupole moment for an oblate ellipsoid $(b<a)$.

For body dimensions comparable but somewhat larger than the penetration depth we can estimate the quadrupole moment assuming charge density $\rho_{-}$within distance $\lambda_{L}$ of the surface and $\rho_{0}$ in the interior. The net

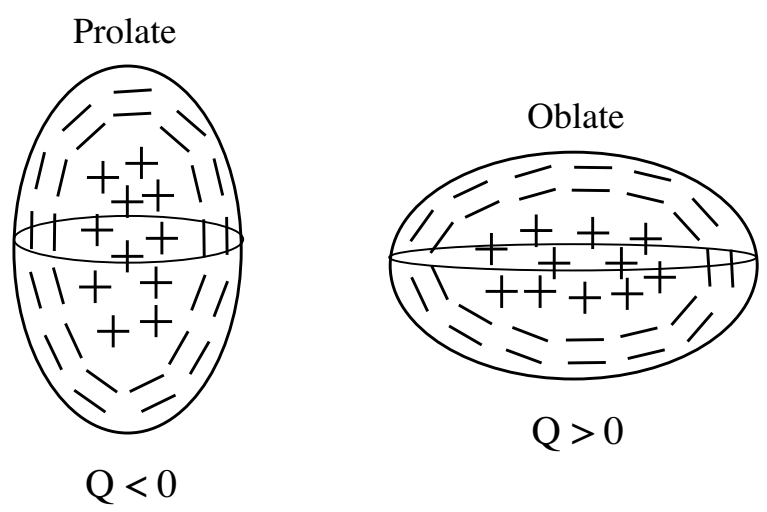

FIG. 3: Charge density in ellipsoids of revolution of dimensions comparable to the penetration depth (schematic). There will be a net quadrupole moment (negative for prolate, positive for oblate) giving rise to an electric field outside the superconductor whose magnitude decays as the fourth power of the distance to the center of the ellipsoid.

quadrupole moment that results is

$$
Q=\frac{16}{15} \pi a^{2} b(b-a) \lambda_{L} \rho_{-}
$$

so that it is negative (positive) for a prolate (oblate) ellipsoid. The electric field at distance $r$ from the center outside the body is of order $E \sim Q / r^{4}$ and should be measurable.

For a body of dimensions much smaller than the London penetration depth we can estimate the net quadrupole moment as follows. The charge density Eq. (30) becomes for the ellipsoid

$$
\rho(r)=\frac{\rho_{0}}{6 \lambda_{L}^{2}}\left[\frac{2 a^{2}+b^{2}}{5}-r^{2}\right]
$$

to ensure charge neutrality, and calculating the integral Eq. (57) over the volume of the ellipsoid yields

$$
Q=-\frac{8}{1575} \pi a^{2} b(b-a)\left(3 a^{3}+3 a^{2} b+4 a b^{2}+4 b^{3}\right) \frac{\rho_{0}}{\lambda_{L}^{2}}
$$

For small distortion from sphericity in terms of $R \sim a \sim b$

$$
Q=-\frac{16}{15} \pi a^{2} b(b-a) \lambda_{L} \times\left[\frac{2}{15} \frac{\rho_{0} R^{3}}{\lambda_{L}^{3}}\right]
$$

which is similar to Eq. (58) for $R \sim \lambda_{L}$. If we relate $\rho_{0}$ to $\rho_{-}$using Eq. (59) with $\rho_{-} \equiv \rho(r=R)$ Eqs. (61) and (58) differ by a factor $4 / 3$.

For body shapes with less symmetry than the ellipsoid, a dipole moment also becomes possible. As an example, consider the body of revolution shown in Figure 4, with radius

$$
r(z)=a+(b-a) \frac{z}{h}
$$




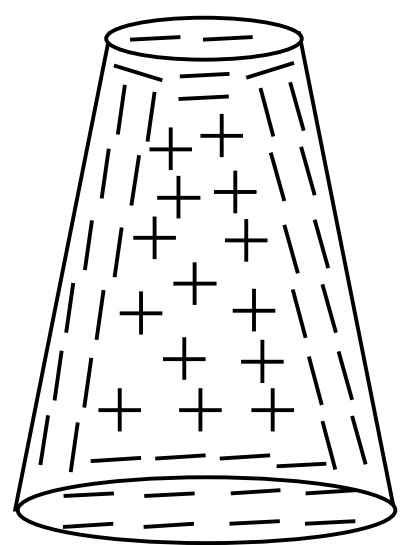

FIG. 4: Charge density in a body of low enough symmetry that it can give rise to an electric dipole moment (schematic). For dimensions of the body comparable to or smaller than the penetration depth there will be a net electric dipole moment along the vertical direction giving rise to a dipolar electric field outside the superconductor.

and $0 \leq z \leq h$. For a uniformly charged such body with charge density $\rho$ the dipole moment relative to $(r=$ $0, z=h / 2)$ is given by

$$
p=\frac{\pi h^{2}}{12}\left(b^{2}-a^{2}\right) \rho
$$

We assume again that the expelled negative charge does not rearrange laterally for body dimensions not much larger than the penetration depth. The net dipole moment is then given by

$$
p=\frac{\pi}{12} \rho_{-} h\left[h\left(b^{2}-a^{2}\right)-h^{\prime}\left(b^{\prime 2}-a^{\prime 2}\right) \frac{a^{2}+a b+b^{2}}{a^{\prime 2}+a^{\prime} b^{\prime}+b^{\prime 2}}\right]
$$

with $h^{\prime}=h-2 \lambda_{L}, a^{\prime}=r\left(\lambda_{L}\right)-\lambda_{L}, b^{\prime}=r\left(h-\lambda_{L}\right)-\lambda_{L}$, and points along the $z$ direction. The sign of $p$ is not simply determined, e.g. for $b<a$ it is generally positive but can become negative for large $h$.

\section{DISCUSSION}

We have proposed that the ground state charge distribution in superconductors is different from what is conventionally assumed. Rather than being uniform and locally charge neutral as normal metals, we propose that the charge distribution in superconductors is inhomogeneous, with more negative charge near the surface and more positive charge in the interior, reflecting the fundamental charge asymmetry of matter.

The charge inhomogeneity is a consequence of the fact that the superfluid electrons are highly mobile and have quantum-mechanical coherence over the macroscopic dimensions of the sample. These electrons have 'undressed' from the electron-ion and the electron-electron interaction [9, 15] and become completely free-electronlike, except for the pairing correlations that bind $k \uparrow$ and $-k \downarrow$ electrons. The quantum-mechanical lowering of kinetic energy of the light electrons due to delocalization causes the electronic charge density to be larger near the boundaries of the sample, just as electrons do not remain confined within the dimensions of the positive nucleus in an ordinary atom.

We argue that the electric field in the interior of superconductors is not screened as in ordinary metals because the ground state wave function has 'rigidity', and the macroscopic quantum-mechanical coherence prevents local deformations that would screen the electric field. As discussed in Sect. 4, an electric field can exist in a superconductor if the superfluid electrons are delocalized over macroscopic distances and their velocity has the proper gradient. At finite temperatures however there are also excited quasiparticles that are not macroscopically coherent, and one may wonder why they do not screen the electric field. We suggest that they are unable to screen the interior field because they are positively charged [16] and as a consequence they are also pushed out towards the surface.

In the Meissner effect, the supercurrents can shield the magnetic field so that it is zero in the interior of the superconductor only if the body's dimensions are larger than the penetration depth; otherwise, the magnetic field penetrates the superconductor. Similarly, we have argued that the negative charge can shield the electric field so that it is zero in the exterior of the superconductor if the body's dimensions are larger than the penetration depth; otherwise, the electric field can leak out from the superconductor to the exterior, unless the body has perfect spherical symmetry.

The latter fact should allow for experimental detection of these electric fields around small superconducting particles of non-spherical shape. Remarkably, very recently Moro et al reported detection of spontaneous electric dipole moments in Nb clusters 17] at low temperatures. Their observations that the effect is strongest for even number of electrons in the cluster and that it occurs only at low temperatures suggests that the effect is related to superconductivity [17] as proposed by the authors. Moro et al concluded from their observations the existence of an internal electric field in the interior of the metal cluster of the order of $10^{6} \mathrm{~V} / \mathrm{cm}$, and stress that such an internal electric field cannot occur in an ordinary metal and suggests a 'rigidity' of the electronic wavefunctions as well as a collective effect. The theory discussed here predicts that dipole moments should arise from clusters of irregular shape such as shown in Fig. 4. Experimentally, relation between the measured dipole moment and the shape of the cluster has not been examined, and it would be interesting to do so to compare with predictions of this theory. Concerning the magnitude of the expected effect, we estimated in Sect. (1) a maximum electric field for $N b$ comparable to the one inferred by Moro et al under the assumption that the energy $\tilde{\epsilon}$ in 
Eq. (7) is the condensation energy. However the magnitude of the electric field will be much smaller for cluster dimensions smaller than the penetration depth according to the results in Sect. III, so that our prediction may be inconsistent with the observation unless $\tilde{\epsilon}$ is assumed to be much larger than the condensation energy.

For small clusters of more regular shape such as ellipsoids of revolution, no dipole moment but a quadrupole moment should be observed, as discussed earlier. Experimental determination of quadrupole moments in superconducting metal clusters has not been reported, and it would be interesting to search for this effect to compare with the theory discussed here. For example, it has been reported that tin clusters adopt prolate geometries [18]. If so, according to the theory discussed here tin clusters at low temperatures should exhibit a negative quadrupole moment.

Further consequences of this physics will be discussed in future work.

\section{Acknowledgments}

The author is grateful to Michel Viret for calling ref. [17] to his attention, and to W.A. de Heer for a stimulating discussion.
[1] J. Bok and J. Klein, Phys.Rev.Lett. 20, 660 (1968).

[2] F. London and H. London, Proc.Roy.Soc. A149, 71 (1935); Physica 2, 341 (1935)

[3] H. London, Proc.Roy.Soc. A155, 102 (1936).

[4] F. London, 'Superfluids', Dover, New York, 1961.

[5] J.E. Hirsch and F. Marsiglio, Phys. Rev. B 39, 11515 ; F. Marsiglio and J.E. Hirsch, Phys. Rev. B41, 6435 (1990).

[6] J.E. Hirsch, Physica C 158, 326 (1989); Physica C 364365, 37 (2001) and references therein.

[7] J.E. Hirsch, Phys.Lett.A 281, 44 (2001).

[8] J.E. Hirsch, Phys.Rev.B 65, 184502 (2002); Phys.Rev.B 68, 012510 (2003).

[9] J.E. Hirsch, Phys.Lett. A 309, 457 (2003); cond-mat/0305574 (2003).

[10] In ref. 9] it was also found that some of the negative charge spills out beyond the surface of the superconductor as indicated in Fig. 1; however that effect will not be considered here.
[11] A. Sommerfeld, "Mechanics of Deformable Bodies", Academic Press, New York, 1971, Chpt. III.

[12] M. von Laue, "Theory of Superconductivity", Academic Press, New York, 1952.

[13] J.D. Jackson, "Classical Electrodynamics", Wiley, New York, 1999.

[14] T. Wangler, in "Principles of RF Linear Accelerators", Wiley, New York, 1998, p. 276.

[15] J.E. Hirsch, Phys.Rev.B 62, 14487 (2000); Phys.Rev.B 62, 14498 (2000).

[16] J.E. Hirsch, Phys. Rev. B 58, 8727 (1998); F. Marsiglio and J.E. Hirsch, Physica C 159, 157 (1989).

[17] R. Moro, X. Xu, S. Yin and W.A. de Heer, Science 300, 1265 (2003).

[18] A.A. Shvartsburg and M.F. Jarrold, Phys. Rev. A 60, 1235 (1999). 